\title{
Threshold Crack Speed Controls Dynamical Fracture of Silicon Single Crystals
}

\author{
Markus J. Buehler, ${ }^{1, *}$ Harvey Tang, ${ }^{2}$ Adri C. T. van Duin, ${ }^{3}$ and William A. Goddard $\mathrm{III}^{3}$ \\ ${ }^{1}$ Laboratory for Atomistic and Molecular Mechanics, Department of Civil and Environmental Engineering, \\ Massachusetts Institute of Technology, 77 Massachusetts Avenue, Room 1-272, Cambridge, Massachusetts 02139, USA \\ ${ }^{2}$ Department of Aeronautical Engineering, Massachusetts Institute of Technology, \\ 77 Massachusetts Avenue, Cambridge, Massachusetts 02139, USA \\ ${ }^{3}$ Division of Chemistry and Chemical Engineering, California Institute of Technology, \\ 1201 E. California Boulevard, Pasadena, California 91125, USA \\ (Received 31 March 2007; published 18 October 2007)
}

Fracture experiments of single silicon crystals reveal that after the critical fracture load is reached, the crack speed jumps from zero to $\approx 2 \mathrm{~km} / \mathrm{sec}$, indicating that crack motion at lower speeds is forbidden. This contradicts classical continuum fracture theories predicting a continuously increasing crack speed with increasing load. Here we show that this threshold crack speed may be due to a localized phase transformation of the silicon lattice from 6-membered rings to a 5-7 double ring at the crack tip.

DOI: 10.1103/PhysRevLett.99.165502

Fracture of solids proceeds by breaking of atomic bonds, leading to formation of new material surfaces. In silicon, this is a complex process that could previously only be captured by quantum mechanics $(\mathrm{QM})[1-4]$, suggesting that fracture in silicon cannot be modeled based on empirical potentials [5-7].

Experiments of fracture of silicon single crystals [7-13] revealed that cracks rapidly jump to a steadystate speed of $\approx 2 \mathrm{~km} / \mathrm{sec}$ at the critical fracture load, while slowly increasing towards $\approx 3.5 \mathrm{~km} / \mathrm{sec}$ for higher loads [13]. This suggests that steady-state crack speeds between zero and $\approx 2 \mathrm{~km} / \mathrm{sec}$ are not allowed, indicating existence of a threshold crack speed. Fracture instabilities occur at crack speeds of $\approx 2 / 3$ of the Rayleigh wave speed $[8,9,14]$, leading to increasingly rough fracture surfaces.

Here we employ a theoretical approach based on a multiparadigm simulation hybrid ReaxFF-Tersoff model (ReaxFF is a force field for reactive systems) [15-18]. We consider a perfect crystal with an initial crack of length $a$ [Fig. 1(a)]. The crystal referred to as the "(111)" system is oriented so that the $x-y-z$ directions are $(11 \overline{1}) \times\left(\frac{1}{2} \times\right.$ $\left.\frac{1}{2} \overline{1}\right) \times\left(\frac{1}{2} \frac{1}{2} 0\right)$, creating a (111) fracture plane with [112] fracture direction. The [e.g., (110)] system is oriented so that the $x-y-z$ directions are $(110) \times(\overline{1} 10) \times(001)$, creating a (110) fracture plane with [110] fracture direction. The initial crack is inserted by removing atoms in a wedgelike shape. We use periodic boundary conditions in the $z$ direction; the thickness of the plane strain system is one unit cell in both orientations. This constraint may influence the possibility of nucleating dislocations; however, this setup represents a good model to study brittle fracture of silicon. While this simulation model does not resemble most experimental fracture specimen geometries, it enables us to gain fundamental insight into the atomistic fracture mechanisms. The crack tip position is determined by finding the surface atom with maximum $y$ position inside a search region [dashed line in Fig. 1(a)], which is averaged to eliminate high frequency fluctuations.
PACS numbers: 62.20.Mk, 46.50.+a

The relaxed fracture surface energies for the (110) and (111) planes are $\gamma_{0,(110)} \approx 1.48 \mathrm{~N} / \mathrm{m}$ and $\gamma_{0,(111)} \approx$ $1.14 \mathrm{~N} / \mathrm{m}$. The value for the (110) surface agrees with experimental results $\gamma_{(110)} \approx 1.7 \mathrm{~N} / \mathrm{m}[9,10]$; for the (111) surface $\gamma_{(111)} \approx 1.1 \mathrm{~N} / \mathrm{m}[13]$. The uniaxial elastic moduli

(a)

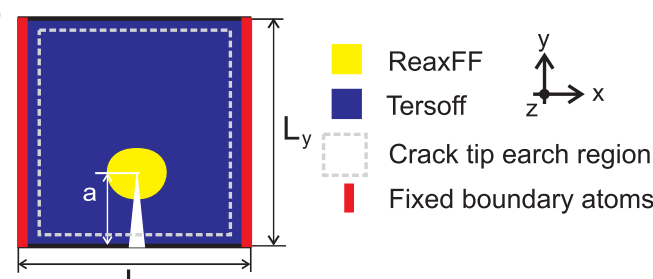

(b)

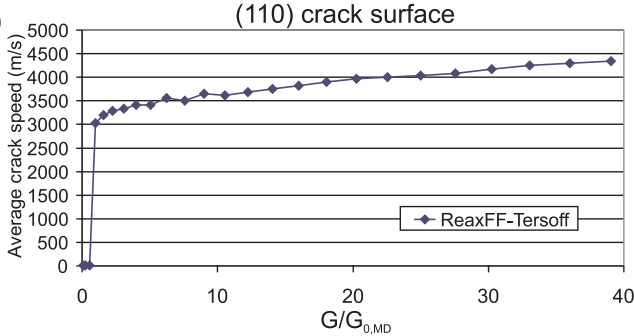

(c)

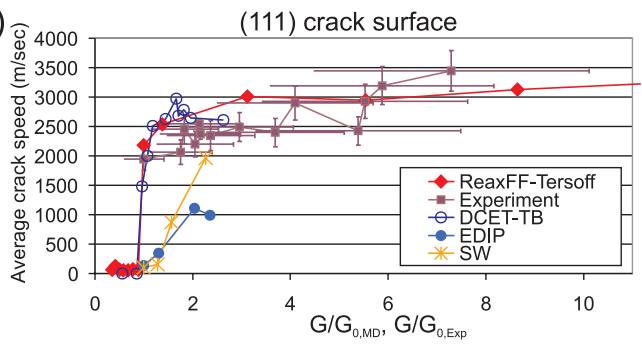

FIG. 1 (color online). Geometry [subplot (a)] and crack speed as a function of reduced load for the (110) system [subplot (b), slab dimensions $56 \mathrm{~nm} \times 80 \mathrm{~nm}$, crack length $a=20 \mathrm{~nm}$, and the (111) system [subplot (c), slab dimensions $26 \mathrm{~nm} \times 46 \mathrm{~nm}$, crack length $a=12 \mathrm{~nm}$ ]. The loading is expressed as the energy release rate, facilitating a quantitative comparison with experiment. 
are $E_{(110)}^{\prime} \approx 2.13 \times 10^{11} \mathrm{~Pa}$ and $E_{(111)}^{\prime} \approx 2.43 \times 10^{11} \mathrm{~Pa}$. The Rayleigh wave speeds are $c_{R,(110)} \approx 4.5 \mathrm{~km} / \mathrm{sec}[8,9]$ and $c_{R,(111)} \approx 4.68 \mathrm{~km} / \mathrm{sec}[3]$. The integration time step $\Delta t=$ $1.5 \mathrm{fs}$.

In the first computational experiment, we apply a fixed load at the beginning of the simulation and hold it constant (temperature $T=300 \mathrm{~K}$, controlled by a Berendsen thermostat [19]). We calculate the steady-state crack speed $v$ as a function of the applied load, expressed in reduced energy release rate $G / G_{0, \mathrm{MD}}[14]$, where $G_{0, \mathrm{MD}}$ is defined as the critical energy release rate at the onset of fracture as determined from molecular dynamics (MD) simulation. The crack speeds are evaluated when the crack is in steady-state propagation, in order to exclude any unrealistic effects in the early stages immediately after the simulation has been started, due to the sudden application of loading (transient effects are damped out by the thermostat).

Figure 1(b) plots $v$ as a function of $G / G_{0, \mathrm{MD}}$ for the (110) system. We find that $v$ remains zero for $G / G_{0, \mathrm{MD}}<$ 1. However, $v$ discontinuously jumps to $\approx 3 \mathrm{~km} / \mathrm{sec}$ for $G / G_{0, \mathrm{MD}} \geq 1$, approaching $\approx 4.5 \mathrm{~km} / \mathrm{sec}$ for higher loads. Figure 1(c) plots similar results for the (111) system. The (111) orientation is identical to that used in recent experimental [13] and other computational studies [3]. This crack dynamics is reminiscent of the lattice-trapping effect $[3,20,21]$, which has also been observed in experiment [13] and simulation [3,21,22].

Figure 1(c) contains a comparison of our results with experimental and earlier computational results, showing that our model quantitatively reproduces experimental results of crack speeds. In contrast, other computational attempts, for example, those using empirical potentials (Stillinger-Weber, Tersoff, EDIP) have failed to reproduce the experimental results qualitatively, as they predict a slower increase of crack speed with increasing $G$ or failed to reproduce the brittle character of silicon [13,15,23,24].

The comparison with experiment shown in Fig. 1(c) has to be considered with care, as the sudden load application is a boundary condition that is significantly different from experimental studies. However, this computational strategy is the best that can be done at this point. The observations made in the simulations contradict predictions of classical linear elastic fracture mechanics (LEFM) [14]: LEFM predicts a continuous increase of $v$ with increasing $G$ [14]. For crack growth resistance $\gamma$, the crack speed is $A\left(v / c_{R}\right) G=\left(1-\frac{v}{c_{R}}\right) G=2 \gamma=G_{0}$. The predicted crack speed is $\frac{v}{c_{R}}=1-\frac{G_{0}}{G}$. Indicating that $v / c_{R}$ is zero at $G=$ $G_{0}=2 \gamma_{0}$ and increases continuously with $G$, predicting that all speeds $0<v<c_{R}$ are admitted. This disagrees our observations [Fig. 1(b) and 1(c)] [13]. Second, we find that the Griffith condition [14] cannot be applied to predict the onset of fracture in silicon. According to the continuumtheory-based Griffith condition applied to a surface crack
$[14,25]$, the critical energy release rate for fracture initiation in a crystal with a surface crack can be estimated by $G=2 \gamma_{0}$.

To test the Griffith condition (which is independent of the specific system geometry for a proper expression of the stress intensity factor $[14,26]$ ), we perform a simulation of a (111) system with dimensions $68 \mathrm{~nm} \times 90 \mathrm{~nm}$ with a small surface crack of $6.8 \mathrm{~nm}$. This geometry corresponds to a system with an infinite array of periodic surface cracks loaded under uniform stress. This is a geometry typically not used in experimental studies; however, this is the best that can be done with our computational method. We carry out a simulation with slowly increasing tensile load (mode I loading $[14,26]$ ), while keeping the system at constant temperature of $300 \mathrm{~K}$ (loading rate $0.00006125 \%$ strain increment per integration step). The critical strain at which the crack starts to propagate is used to extract the critical applied fracture stress $\sigma_{0, \mathrm{MD}}$. Based on this estimate, the stress intensity factor (SIF) for the crack in this model is then approximated by $K_{I} \approx 1.12 \sqrt{\pi a} \sigma_{0}$, where $\sigma_{0}$ is the applied tensile stress [14]. This surface crack model is a reasonable approximation since $a \ll\left\{L_{x}, L_{y}\right\}$ [27]. The parameter $G=K_{I}^{2} / E^{\prime}$ is the energy release rate corresponding to the applied SIF $K_{I}$ [14] $G=1.12^{2} \sigma_{0}^{2} \pi a / E^{12}$. Our simulations suggest that the critical energy release rate for the (111) orientation is $G_{0, \mathrm{MD}}=5 \mathrm{~N} / \mathrm{m}$. This value is 2.2 times higher than the theoretical prediction based on $G=$ $\gamma_{\mathrm{O}_{2}}$. The particular boundary conditions used in the MD simulations are different than those used in most experiments.

These results hint that there exist differences between the LEFM model and the MD results. In comparable studies with a simple pair potential between two harmonic half-spaces, it has been shown that the Griffith model is a reasonable approximation [27]. This indicates that the different behavior of silicon may be due to more complex, valency induced atomistic processes at the crack tip in silicon.

To investigate the atomistic details of the fracture dynamics in silicon, we analyze a computational experiment with a slowly increasing tensile load (mode I load [14,26]) for the (111) oriented crystal (strain rate $0.0005 \%$ strain increment per integration step). Figure 2 depicts snapshots for a crack oriented in the (111) plane. The initial static regime is followed by a short period of crack growth during which a perfectly flat, mirrorlike surface is generated. Crack propagation becomes increasingly erratic. Figure 3 depicts an analysis of the sequence of atomic events. The first single bond rupture event is due to a local rearrangement of the atoms. After this initial event, further crack extension does not occur even though the load is increased. Instead of crack extension, we observe a change in the local crystal structure at the tip of the crack. Two of the 6membered silicon rings transform into a 5-7 double ring combination, where the 7-membered ring is closer to the 
(a) $t=3 \mathrm{ps}$

(b) $t=4.2 \mathrm{ps}$
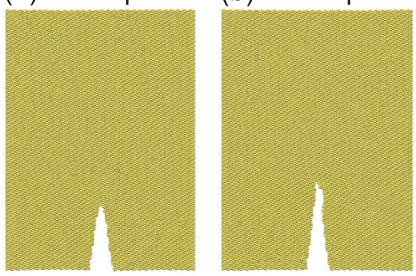

(c) $t=4.65 \mathrm{ps}$

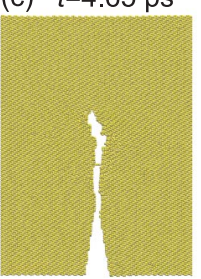

(d) $t=5.25 \mathrm{ps}$

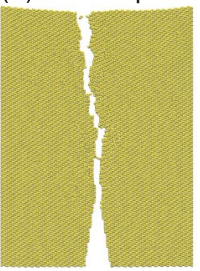

FIG. 2 (color online). Snapshots of crack propagation in a perfect single crystal of silicon for the (111) crack orientation, mode I loading.

tip of the crack [see Fig. 3(b)]. Creation of this defect appears to be induced by the increased stresses in the vicinity of the crack tip. Formation of the 5-7 double ring has also been observed in tight-binding (TB) approximate QM calculations under similar loading conditions [28].

Crack nucleation after the 5-7 double ring has been formed occurs not at the primary crack tip. Instead, a small secondary microcrack forms ahead of the primary crack [Fig. 3(c)], which reunites with the primary crack [Fig. 3(d)].

Subsequently, the crack begins to propagate at a speed close to $2 \mathrm{~km} / \mathrm{sec}$. This minimum crack speed coincides with the smallest admissible speed observed under constant load [see Fig. 1(c)]. We have confirmed this behavior at various pulling rates.

Figure 4 depicts the crack tip velocity history and the onset of the crack tip instability.

The threshold crack speed may be related to formation of the 5-7 double ring. Two observations support this hypothesis. First, there exists a geometric effect due to crack blunting, effectively leading to a reduction of the stress concentration at the tip of the crack [compare Figs. 3(a) and 3(b)]. In this spirit, the 5-7 defect corresponds to the fracture process zone; since it is only a few atomic distances wide and much smaller than the specimen

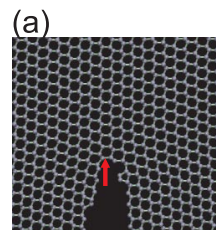

(d)

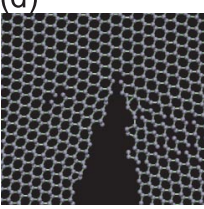

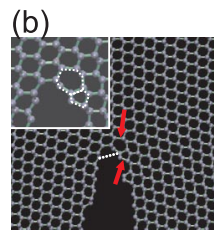

(e)

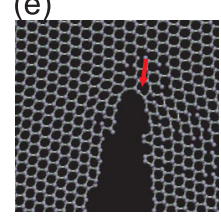

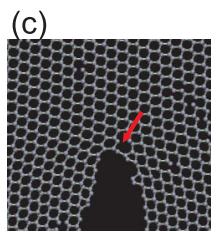

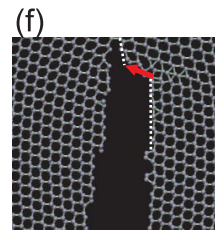

FIG. 3 (color online). Snapshots of dynamical crack propagation in silicon, showing formation of a 5-7-ring combination [subplot (b)]. Subplots (a), (b) are taken at $t_{A}=0.08$ ps and $t_{C}=1.58 \mathrm{ps}$; for other times see Fig. 4 . dimension, the small-scale yielding condition is satisfied [14]. Second, the 5-7 double ring effectively leads to an increased energy barrier for crack nucleation. The apparent fracture surface energy is increased to $\gamma_{5-7}>\gamma_{0}$. After the load is sufficient to break the 5-7 double ring, the crack propagates continuously without formation of the 5-7 double ring, while the crack senses a fracture resistance according to $\gamma_{0}$. We estimate $\gamma_{5-7} \approx 2.5 \mathrm{~N} / \mathrm{m}$, leading to a fracture toughness of $K_{\mathrm{Ic}, \mathrm{MD}} \approx 1.1 \mathrm{MPa} \sqrt{m}$, which is close to experimental measurements suggesting $K_{\mathrm{Ic}} \approx$ $0.82-1.23 \mathrm{MPa} \sqrt{m}$ for the same crystal orientation [12]. This agreement provides additional evidence that supports our hypothesis. Estimates of the fracture toughness purely based on the (111) surface energy lead to values of $K_{\text {Ic }}$ that are approximately $30 \%$ smaller $\left(K_{\mathrm{Ic}} \approx 0.76 \mathrm{MPa} \sqrt{m}\right)$ $[12,29]$. Notably, this value does not fall into the range of experimental measurements [12].

Additional evidence exists that formation of the 5-7 double ring defect is associated with an increase of resistance to crack extension. The formation of 5-7 defects induces the crack instability shown in Fig. 3(f). Once the defect is formed, the crack changes direction to "avoid" breaking the 5-7 double ring defect. The increase of resistance is reflected in a sudden drop of the crack speed, as shown in Fig. 4. The crack instability sets in at $\approx 69 \%$ of the Rayleigh-wave speed, in qualitative agreement with experimental results $[8,9]$. Both results indicate that crack instabilities begin at larger speeds than $1 / 3 c_{R}$, the dynamical crack tip instability speed found in many materials [30-32]. This may be due to the fact that $v<2 \mathrm{~km} / \mathrm{sec}$ is not admissible in silicon and thus alternative instability mechanisms dominate.

The jump of crack speed to a finite value can be explained based on a simple consideration of the energy balance. Changes in $\gamma$ lead to immediate changes in the crack speed [14,33]: fracture initiation is controlled by the apparent surface energy $\gamma=\gamma_{5-7}$. However, after the critical load is reached, crack dynamics is controlled by continuous creation of (111) surfaces, characterized by $\gamma_{0}<\gamma_{5-7}$ and thus fracture resistance will lead to a finite crack speed immediately after crack nucleation. The crack speed is

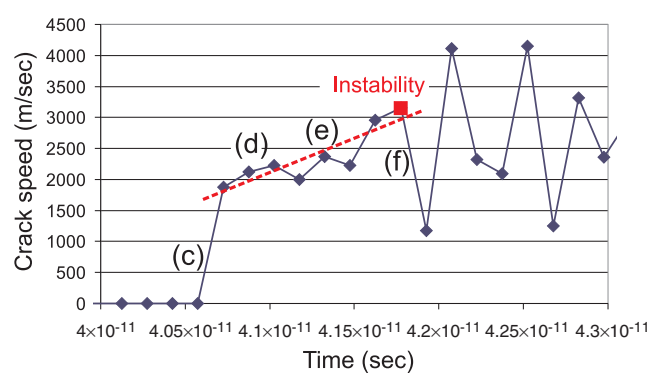

FIG. 4 (color online). Velocity-time history of the crack dynamics shown in Fig. 3. 


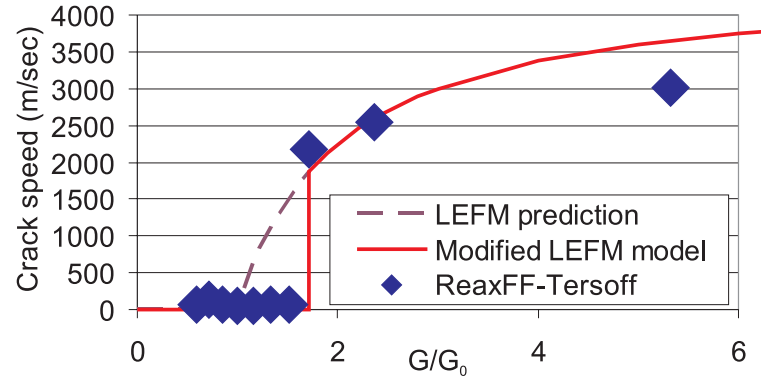

FIG. 5 (color online). Comparison of the prediction of LEFM, modified LEFM [Eq. (1)], and MD simulation. Results are plotted as a function of $G / G_{0, \mathrm{MD} / \mathrm{Exp} \text {. }}$

$$
\frac{v_{0}}{c_{R}}(G)=\left\{\begin{array}{ll}
0 & G<G_{0, \mathrm{MD}} \\
1-\frac{G_{0}}{G} & G \geq G_{0, \mathrm{MD}}
\end{array},\right.
$$

where $G$ is the applied energy release rate, $G_{0}$ the critical energy release rate [according to the (111) surface energy $\left.\gamma_{0}\right]$, and $G_{0, \mathrm{MD}}$ the critical energy release at which fracture actually occurs (due to the fracture surface energy $\gamma_{5-7}$ ). The crack speed at the critical load $G=G_{0, \mathrm{MD}}$ is $c_{R}(1-$ $\left.G_{0} / G_{0, \mathrm{MD}}\right)>0$ while $G_{0} / G_{0, \mathrm{MD}}<1$. The admissible crack speeds are $c_{R}\left(1-G_{0} / G_{0, \mathrm{MD}}\right) \leq v_{0}<c_{R}$. This is in contrast to the prediction by LEFM [14,25] suggesting that $0 \leq v_{0}<c_{R}$ under identical boundary conditions. Figure 5 shows a quantitative comparison of LEFM, modified LEFM, and MD. Our model predicts an initial crack speed of $\approx 2.5 \mathrm{~km} / \mathrm{sec}$ for the (111) orientation, consistent with our observations [Fig. 1(b) and 1(c)]. We note that this result agrees quantitatively with earlier simulations [22]. The modified LEFM model overpredicts crack speeds for large $G$. A possible reason for this disagreement could be elastic softening that leads to a reduction of $c_{R}[5,6,22]$.

The results show that in addition to instability driving forces such as energy flow and change of asymptotic stress field $[5,6,32,34]$, chemical rearrangements may contribute to fracture instabilities, suggesting an intimate connection between fracture mechanics and chemistry.

Similar mechanisms as in silicon may occur in other covalently bonded materials. A wider implication of this study is that properties such as the fracture surface energy are not material parameters alone, but may depend on the physical state of the system.

H. T. acknowledges support from MIT's UROP program. Funding was provided by ARO (Grant No. W911NF-06-1-0291).

*Corresponding author. mbuehler@MIT.EDU

[1] R. D. Deegan et al., Phys. Rev. E 67, 066209 (2003).
[2] A. D. Becke, J. Chem. Phys. 98, 5648 (1993).

[3] N. Bernstein and D. W. Hess, Phys. Rev. Lett. 91, 025501 (2003).

[4] N. Bernstein and D. W. Hess, MRS Symposia Proceedings, edited by L. P. Kubin, R. L. Selinger, J.L. Bassani, and K. Cho (MRS, Warrendale, PA, 2000), Vol. 653, p. Z2.7.1.

[5] M.J. Buehler and H. Gao, Nature (London) 439, 307 (2006).

[6] M.J. Buehler, F.F. Abraham, and H. Gao, Nature (London) 426, 141 (2003).

[7] D. Holland and M. Marder, Phys. Rev. Lett. 80, 746 (1998).

[8] T. Cramer, A. Wanner, and P. Gumbsch, Phys. Status Solidi A 164, R5 (1997).

[9] T. Cramer, A. Wanner, and P. Gumbsch, Phys. Rev. Lett. 85, 788 (2000).

[10] R. F. Cook, J. Mater. Sci. 41, 841 (2006).

[11] E. D. Renuart et al., J. Mater. Res. 19, 2635 (2004).

[12] A. M. Fitzgerald et al., J. Mater. Res. 17, 683 (2002).

[13] J. A. Hauch et al., Phys. Rev. Lett. 82, 3823 (1999).

[14] L. B. Freund, Dynamic Fracture Mechanics (Cambridge University Press, Cambridge, England, 1990).

[15] M. J. Buehler, A.C.T. van Duin, and W. A. Goddard, Phys. Rev. Lett. 96, 095505 (2006).

[16] A. C. T. v. Duin et al., J. Phys. Chem. A 105, 9396 (2001).

[17] A. C. T. v. Duin et al., J. Phys. Chem. A 107, 3803 (2003).

[18] J. Tersoff, Phys. Rev. Lett. 61, 2879 (1988).

[19] M.P. Allen and D. J. Tildesley, Computer Simulation of Liquids (Oxford University Press, New York, 1989).

[20] T. Zhu, J. Li, and S. Yip, Proc. R. Soc. A 462, 1741 (2006).

[21] R. Perez and P. Gumbsch, Phys. Rev. Lett. 84, 5347 (2000).

[22] J. G. Swadener, M. I. Baskes, and M. Nastasi, Phys. Rev. Lett. 89, 085503 (2002).

[23] M.Z. Bazant, E. Kaxiras, and J. F. Justo, Phys. Rev. B 56, 8542 (1997).

[24] F. H. Stillinger and T. A. Weber, Phys. Rev. B 31, 5262 (1985).

[25] T.L. Anderson, Fracture Mechanics: Fundamentals and Applications (CRC Press, Boca Raton, FL, 1991).

[26] K. B. Broberg, Cracks and Fracture (Academic, New York, 1990).

[27] H. Tada, P. C. Paris, and G. R. Irwin, The Stress Analysis of Cracks Handbook (ASME Press, New York, 2000).

[28] G. Szanyi, personal communication. [Similar 6-6 to 5-7 transformations as observed in our studies have been observed in TB calculations (similar geometry).]

[29] A. M. Fitzgerald, R. H. Dauskardt, and T. W. Kenny, Sens. Actuators A, Phys. 83, 194 (2000).

[30] J. Fineberg et al., Phys. Rev. Lett. 67, 457 (1991).

[31] J. Fineberg et al., Phys. Rev. B 45, 5146 (1992).

[32] H. Gao, J. Mech. Phys. Solids 44, 1453 (1996).

[33] M. J. Buehler, H. Gao, and Y. Huang, Comput. Mater. Sci. 28, 385 (2003).

[34] M.J. Buehler, F.F. Abraham, and H. Gao, Springer Lecture Notes in Computational Science and Engineering (Springer, New York, 2004), p. 143. 